PROCEEDINGS OF THE

AMERICAN MATHEMATICAL SOCIETY

Volume 126, Number 6, June 1998, Pages 1769-1782

S $0002-9939(98) 04256-7$

\title{
BOUNDED SEQUENCE-TO-FUNCTION GENERALIZED HAUSDORFF TRANSFORMATIONS
}

\author{
B. E. RHOADES
}

(Communicated by Christopher D. Sogge)

ABstRaCt. Georgakis (1988) obtained the norm of the transformation

$$
(T a)(y)=\sum_{n=0}^{\infty}(-y)^{n} \frac{g^{(n)}(y)}{n !} a_{n}, \quad y \geq 0,
$$

considered as an operator from the sequence space $\ell^{p}$, with weights $\Gamma(n+s+1) / n$ ! to $L^{p}[0, \infty)$, with weight $y^{s}, s>-1$. As corollaries he obtained inequality statements for Borel and generalized Abel transformations. He also obtained the best constants possible for several weighted norm inequalities of Hardy and Littlewood. In this paper Georgakis' results are extended to the Endl generalized Hausdorff matrices.

Let $\left\{\mu_{n}\right\}$ denote a real or complex sequence. The difference operator $\Delta$ is defined by $\Delta \mu_{k}=\mu_{k}-\mu_{k+1}, \Delta^{n+1} \mu_{k}=\Delta\left(\Delta^{n} \mu_{k}\right)$. The Endl generalized Hausdorff matrices, developed independently by Endl [1] and Jakimovski [4], are lower triangular matrices with nonzero entries $\left(\begin{array}{l}n+\alpha \\ n-k\end{array}\right) \Delta^{n-k} \mu_{k}, 0 \leq k \leq n, \alpha \geq 0$.

From [4], page 22, the Endl Hausdorff transformation generated by a completely monotone function $g$, or, what is equivalent, the Laplace transform of a finite positive Borel measure $\sigma$ on $[0, \infty)$, is given by

$$
T a(y)=\sum_{n=0}^{\infty} \frac{(-1)^{n}(y+\alpha)^{n+\alpha} g^{(n)}(y)}{\Gamma(n+\alpha+1)} a_{n},
$$

where

$$
g(y):=\int_{0}^{\infty} e^{-t(y+\alpha)} d \sigma(t)
$$

Then

$$
(T a)(y)=\sum_{n=0}^{\infty} a_{n} \int_{0}^{\infty} \frac{(y+\alpha)^{\alpha}(t(y+\alpha))^{n}}{\Gamma(n+\alpha+1)} e^{-t(y+\alpha)} d \sigma(t) .
$$

Received by the editors March 9, 1995 and, in revised form, December 6, 1996.

1991 Mathematics Subject Classification. Primary 40C10, 40G05.

Key words and phrases. Generalized Hausdorff, transformation, bounded operator, weighted $\ell^{p}$-spaces.

(C)1998 American Mathematical Society 
Let $\ell^{p, s}$ and $L^{p, s}[0, \infty)$ denote, respectively, the space of sequences $a=\left\{a_{n}\right\}$ and functions $f(y), y \geq 0$, such that

$$
\begin{aligned}
\|a\|_{p, s}^{p} & :=\sum_{n=0}^{\infty}\left|a_{n}\right|^{p}(n+\alpha+1)_{s}<\infty, \\
\|f\|_{p, s}^{p} & :=\int_{0}^{\infty}|f(y)|^{p}(y+\alpha)^{s} d y<\infty,
\end{aligned}
$$

where $(n+\alpha+1)_{s}:=\Gamma(n+\alpha+s+1) / \Gamma(n+\alpha+1)$. Let $p^{\prime}$ denote the conjugate index of $p$.

Theorem 1. Let $\sigma$ be a positive Borel measure of finite variation on $[0, \infty)$ such that $\sigma(\{0\})=0$ and $T$ be the transformation given by (1), where

$$
C(p, s):=\int_{0}^{\infty} t^{-\alpha-(1+s) / p} d|\sigma|(t), \quad s>-1, \alpha \geq 0,
$$

and $a$ is a sequence in $\ell^{p, s}$. Then (a) $T$ is a bounded transformation of $\ell^{p, s}$ into $L^{p, s}[0, \infty)$ and

$$
\|T a\|_{p, s} \leq C(p, s)\|a\|_{p, s}
$$

for $1 \leq p \leq \infty$. (b) (i) $\|T a\|_{p, s}^{p}<C(p, s)\|a\|_{p, s}^{p}$ for $1<p<\infty$ unless the sequence $\left\{a_{n}\right\}$ is null; (ii) $\|T\|=C(p, s)$ for $1 \leq p \leq \infty$; (iii) The condition $C(p, s)<\infty$ is also necessary for $T$ to be bounded.

Proof. For $p=\infty$ the result is clearly true, and for $p=1$, it follows by Fubini's theorem. Let $1<p<\infty, a \in \ell^{p, s}$, and define the transformation $E_{t}, t>0$, by

$$
\begin{aligned}
\left(E_{t} a\right)(y)= & \sum_{n=0}^{\infty} \frac{(t(y+\alpha))^{n+\alpha}}{t^{\alpha} \Gamma(n+\alpha+1)} e^{-t(y+\alpha)}\left|a_{n}\right| \\
= & \frac{1}{t^{\alpha}} \sum_{n=0}^{\infty}\left(\frac{(t(y+\alpha))^{n+\alpha}}{\Gamma(n+\alpha+1)} e^{-t(y+\alpha)}\left|a_{n}\right|^{p}\right)^{1 / p}\left(\frac{(t(y+\alpha))^{n+\alpha}}{\Gamma(n+\alpha+1)} e^{-t(y+\alpha)}\right)^{1 / p^{\prime}} \\
\leq & \frac{1}{t^{\alpha}}\left(\sum_{n=0}^{\infty} \frac{(t(y+\alpha))^{n+\alpha}}{\Gamma(n+\alpha+1)} e^{-t(y+\alpha)}\left|a_{n}\right|^{p}\right)^{1 / p} \\
& \cdot\left(\sum_{n=0}^{\infty} \frac{(t(y+\alpha))^{n+\alpha}}{\Gamma(n+\alpha+1)} e^{-t(y+\alpha)}\right)^{1 / p^{\prime}}
\end{aligned}
$$

Define

$$
h(u)=\sum_{n=0}^{\infty} \frac{u^{n+\alpha}}{\Gamma(n+\alpha+1)} .
$$

Then $h^{\prime}(u)=u^{\alpha-1} / \Gamma(\alpha)+h(u)$, which is a first order linear differential equation with general solution

$$
h(u)=e^{u} \int_{0}^{u} \frac{x^{\alpha-1} e^{-x}}{\Gamma(\alpha)} d x+c e^{u} .
$$


Since $h(0)=0$ for $\alpha>0, c=0$ and $h(u) \leq e^{u}$. Therefore

$$
\sum_{n=0}^{\infty} \frac{(t(y+\alpha))^{n+\alpha}}{\Gamma(n+\alpha+1)} e^{-t(y+\alpha)} \leq 1,
$$

and

$$
\left(E_{t} a\right)(y) \leq \frac{1}{t^{\alpha}}\left(\sum_{n=0}^{\infty} \frac{(t(y+\alpha))^{n+\alpha}}{\Gamma(n+\alpha+1)} e^{-t(y+\alpha)}\left|a_{n}\right|^{p}\right)^{1 / p} .
$$

If $t>0, y>-\alpha$, and we have equality in (5), then

$$
\begin{aligned}
& \left|a_{n}\right|^{p}(t(y+\alpha))^{n+\alpha} e^{-t(y+\alpha)} / \Gamma(n+\alpha+1) \\
& \quad=M(t(y+\alpha))^{n+\alpha} e^{-t(y+\alpha)} / \Gamma(n+\alpha+1)
\end{aligned}
$$

for $n \geq 0$, and for some positive constant $M$. This implies that $\left|a_{n}\right|^{p}=M$ for $n \geq 0$, and hence that $\|a\|_{p, s}^{p}=M^{p} \sum_{n=0}^{\infty}(n+\alpha+1)_{s}$, which is divergent for $s>-1$, a contradiction. Therefore we have strict inequality, unless $a$ is a null sequence.

Now

$$
\begin{aligned}
&|(T a)(f)|=\left|\int_{0}^{\infty}(T a)(y) f(y)(y+\alpha)^{s} d y\right| \\
& \leq \int_{0}^{\infty}|f(y)|(y+\alpha)^{s} d y \int_{0}^{\infty}\left(E_{t} a\right)(y) d|\sigma|(t) \\
& \leq \int_{0}^{\infty} \int_{0}^{\infty}|f(y)|(y+\alpha)^{s} \frac{1}{t^{\alpha}}\left(\sum_{n=0}^{\infty} \frac{(t(y+\alpha))^{n+\alpha}}{\Gamma(n+\alpha+1)} e^{-t(y+\alpha)}\left|a_{n}\right|^{p}\right)^{1 / p} d|\sigma|(t) d y \\
& \leq {\left[\int_{0}^{\infty} \int_{0}^{\infty} \sum_{n=0}^{\infty}\left|a_{n}\right|^{p} e^{-t(y+\alpha)} t^{-\alpha+(1+s) / p^{\prime}} \frac{(t(y+\alpha))^{n+\alpha}}{\Gamma(n+\alpha+1)}(y+\alpha)^{s} d|\sigma|(t) d y\right]^{1 / p} } \\
& \quad\left[\int_{0}^{\infty} \int_{0}^{\infty}|f(y)|^{p^{\prime}} t^{-\alpha-(1+s) / p}(y+\alpha)^{s} d|\sigma|(t) d y\right]^{1 / p^{\prime}}=A \times B,
\end{aligned}
$$

say.

$$
\begin{gathered}
B=\left[\int_{0}^{\infty}|f(y)|^{p^{\prime}}(y+\alpha)^{s} d y \int_{0}^{\infty} t^{-\alpha-(1+s) / p} d|\sigma|(t)\right]^{1 / p^{\prime}}=\|f\|_{p^{\prime}, s}(C(p, s))^{1 / p^{\prime}} . \\
A=\left[\int_{0}^{\infty} t^{-\alpha+(1+s) / p^{\prime}} \sum_{n=0}^{\infty} \frac{\left|a_{n}\right|^{p}}{\Gamma(n+\alpha+1)}\right. \\
\left.\cdot\left\{\int_{0}^{\infty}(t(y+\alpha))^{n+\alpha} e^{-t(y+\alpha)}(y+\alpha)^{s} d y\right\} d|\sigma|(t)\right]^{1 / p} .
\end{gathered}
$$

The quantity in braces is dominated by $t^{-s-1} \Gamma(n+\alpha+s+1)$. Thus

$$
A \leq\left[\sum_{n=0}^{\infty}(n+\alpha+1)_{s}\left|a_{n}\right|^{p} \int_{0}^{\infty} t^{-\alpha+(1+s)\left(1 / p^{\prime}-1\right)} d|\sigma|(t)\right]^{1 / p}=\|a\|_{p, s}(C(p, s))^{1 / p} .
$$


Therefore

$$
|(T a)(f)| \leq C(p, s)\|a\|_{p, s}\|f\|_{p^{\prime}, s},
$$

and (3) is satisfied.

Now let $a_{n}:=(n+\alpha+s+1)^{-1 / p-\varepsilon}(n+\alpha+1)_{s}^{-1 / p}, 1>\varepsilon>0, s>-1, \alpha \geq 0$.

$$
\begin{aligned}
\|a\|_{p, s}^{p} & =\sum_{n=0}^{\infty}(n+\alpha+1)_{s}(n+\alpha+1)_{s}^{-1}(n+\alpha+s+1)^{-1 / p-\varepsilon} \\
& =\sum_{n=0}^{\infty}(n+\alpha+s+1)^{-1 / p-\varepsilon} .
\end{aligned}
$$

Lemma 1. (a) If $u, v>0$, then

$$
\Gamma(u+v) / \Gamma(u) \begin{cases}\leq u^{v} & \text { for } 0<v \leq 1, \text { and } \\ \geq u^{v} & \text { for } v>1\end{cases}
$$

(b) $(n+\alpha+1)_{s} \leq(n+\alpha+1)^{s+1} /(n+\alpha+s+1)$ for $n \geq 0,-1<s<0$,

(c) $(n+\alpha+1)_{s} \leq(n+\alpha+s+1)^{s}$ for $n \geq 0, s \geq 0$.

Part (a) is part (a) of Lemma 1 of [2]. The other parts are proved in the same way as their counterparts in [2].

From Lemma 1, for $-1<s<0$,

$$
\begin{aligned}
a_{n} & \geq(n+\alpha+s+1)^{-1 / p-\varepsilon}\left(\frac{n+\alpha+s+1}{(n+\alpha+1)^{s+1}}\right)^{1 / p} \\
& =(n+\alpha+s+1)^{-\varepsilon}(n+\alpha+1)^{-(s+1) / p} \geq(n+\alpha+1)^{-(s+1) / p-\varepsilon} .
\end{aligned}
$$

Again from Lemma 1 , for $s \geq 0$,

$$
a_{n} \geq(n+\alpha+s+1)^{-\varepsilon-(s+1) / p} .
$$

Define $s^{*}=\max (s, 0), \gamma=1 / \Gamma((1+s) / p+\varepsilon)$. Then

$$
\begin{gathered}
a_{n} \geq\left(n+\alpha+s^{*}+1\right)^{-\varepsilon-(s+1) / p}=\gamma \int_{0}^{\infty} x^{(1+s) / p+\varepsilon-1} e^{-\left(n+\alpha+s^{*}+1\right) x} d x . \\
(T a)(y) \geq \gamma \int_{0}^{\infty} \frac{d \sigma(t)}{t^{\alpha}} \int_{0}^{\infty} x^{(1+s) / p+\varepsilon-1} \\
\cdot\left[\sum_{n=0}^{\infty} e^{-t(y+\alpha)} \frac{(t(y+\alpha))^{n+\alpha}}{\Gamma(n+\alpha+1)} e^{-\left(n+\alpha+s^{*}+1\right) x}\right] d x .
\end{gathered}
$$

Using (4),

$$
\sum_{n=0}^{\infty} \frac{(t(y+\alpha))^{n+\alpha} e^{-(n+\alpha) x}}{\Gamma(n+\alpha+1)}=e^{t(y+\alpha) e^{-x}} \int_{0}^{t(y+\alpha) e^{-x}} \frac{u^{\alpha-1} e^{-u}}{\Gamma(\alpha)} d u .
$$

Then, since $e^{-x}-1>x$ for $x>0$,

$$
\begin{aligned}
\|T a\|_{p, s}^{p} \geq & \int_{0}^{\infty}(y+\alpha)^{s}\left[\gamma \int_{0}^{\infty} \int_{0}^{\infty} x^{(1+s) / p+\varepsilon-1} e^{-\left(s^{*}+1+t(y+\alpha) x\right)} x\right. \\
& \left.\times \int_{0}^{t(y+\alpha) e^{-x}} \frac{u^{-\alpha-1}}{\Gamma(\alpha)} d u d x \frac{d \sigma(t)}{t^{\alpha}}\right] d y
\end{aligned}
$$


By the mean value theorem for integrals, there exists a number $\xi, 0<\xi<\infty$, such that

$$
\begin{aligned}
I_{1}: & =\gamma \int_{0}^{\infty} \int_{0}^{\infty} x^{(1+s) / p+\varepsilon-1} e^{-\left(s^{*}+1+t(y+\alpha)\right) x} \int_{0}^{t(y+\alpha) e^{-\xi}} \frac{u^{-\alpha-1}}{\Gamma(\alpha)} d u d x \frac{d \sigma(t)}{t^{\alpha}} \\
& =\gamma \int_{0}^{\infty} \int_{0}^{\infty} x^{(1+s) / p+\varepsilon-1} e^{-\left(s^{*}+1+t(y+\alpha)\right) x} \int_{0}^{t(y+\alpha) e^{-\xi}} \frac{u^{-\alpha-1}}{\Gamma(\alpha)} d u d x \frac{d \sigma(t)}{t^{\alpha}} \\
& =\int_{0}^{\infty} \int_{0}^{t(y+\alpha) e^{-\xi}} \frac{u^{\alpha-1} e^{-u}}{\Gamma(\alpha)} d u\left(s^{*}+1+t(y+\alpha)\right)^{-(1+s) / p-\varepsilon} \frac{d \sigma(t)}{t^{\alpha}} .
\end{aligned}
$$

Again by the mean value theorem for integrals there exists a number $\zeta, 0<\zeta<\infty$, such that

$$
I_{1}=\int_{0}^{\zeta(y+\alpha) e^{-\xi}} \frac{u^{\alpha-1} e^{-u}}{\Gamma(\alpha)} d u \int_{0}^{\infty} t^{-\alpha}\left(s^{*}+1+t(y+\alpha)\right)^{-(1+s) / p-\varepsilon} d \sigma(t) .
$$

Since $\xi$ and $\eta$ depend on the variables $x$ and $t$, it is necessary to show that

$$
A:=\int_{0}^{\zeta(y+\alpha) e^{-\xi}} \frac{u^{\alpha-1} e^{-u}}{\Gamma(\alpha)} d u
$$

is bounded away from zero, independent of $\varepsilon$. To this end it will be sufficient to show that $I_{1}$ is uniformly bounded away from zero, independent of $\varepsilon$.

Since $\sigma$ is a positive measure, either there exists an interval $[\beta, \gamma]$ over which $\sigma$ is positive, or $\sigma$ consists of point masses.

Define

$$
f(x, t, y)=t^{-\alpha} x^{(1+s) / p+\varepsilon-1} e^{-\left(s^{*}+1+t(y+\alpha)\right) x} \int_{0}^{t(y+\alpha) e^{-x}} \frac{u^{\alpha-1} e^{-u}}{\Gamma(\alpha)} d u .
$$

Since the integrand is positive, there exists a rectangle $R=[\beta, \gamma] \times\left[x_{1}, x_{2}\right]$, throughout which $f$ is bounded away from zero. By reducing the size of the interval, if necessary, we may assume that either $x_{2} \leq 1$ or $x_{1} \geq 1$. In the first case replace $x^{(1+s) / p+\varepsilon-1}$ by $x^{(1+s) / p-1}$, and in the second replace it by $x^{(1+s) / p-2}$. In either case call the resulting function $g(x, t, y)$.

Then

$$
\begin{aligned}
I_{1} & >\gamma \int_{\beta}^{\gamma} \int_{x_{1}}^{x_{2}} g(x, t, y) d u d x \\
& >\gamma(\gamma-\beta)\left(x_{2}-x_{1}\right) m,
\end{aligned}
$$

where $m$ is the minimum of $g$ over $R$.

If $\sigma$ consists of point masses, simply select one that is positive, and repeat the above argument. It cannot be the case that $\sigma$ is a point mass concentrated at zero, for this would contradict (2).

Let $B=\sup _{\xi} \inf _{\zeta} \inf _{\varepsilon>0} A$. By the above argument $B>0$.

For any $N \geq 2$,

$$
\|T a\|_{p, s}^{p} \geq \int_{N+s}^{\infty} B^{p}(y+\alpha)^{s}\left[\int_{1 /(N+s)}^{\infty}\left(s^{*}+1+t(y+\alpha)\right)^{-(1+s) / p-\varepsilon} \frac{d \sigma(t)}{t^{\alpha}}\right]^{p} d y
$$


Fix $0<\eta<1$. Since $B$ is an increasing function of $y$ choose $N$ large enough so that $B>1-\eta$. Then

$$
\begin{gathered}
\|T a\|_{p, s}^{p} \geq(1-\eta)^{p} \int_{N+s}^{\infty}(y+\alpha)^{-1-\varepsilon p} \\
\cdot\left[\int_{1 /(N+s)}^{\infty}\left(\frac{s^{*}+1}{y+\alpha}+t\right)^{-(1+s) / p-\varepsilon} t^{-\alpha} d \sigma(t)\right]^{p} d y \\
\geq(1-\eta)^{p} \int_{N+s}^{\infty}(y+\alpha)^{-1-\varepsilon p} \\
\geq(1-\eta)^{p} \sum_{n=N}^{\infty}(n+\alpha+s+1)^{-(1+\varepsilon p)} \cdot\left[\int_{1 /(N+s)}^{\infty}\left(\frac{s^{*}+1}{N+s+\alpha}+t\right)^{-(1+s) / p-\varepsilon} t^{-\alpha} d \sigma(t)\right]^{p} d y \\
\cdot\left[\int_{1 /(N+s)}^{\infty}\left(\frac{s^{*}+1}{N+s+\alpha}+t\right)^{-(1+s) / p-\varepsilon} t^{-\alpha} d \sigma(t)\right]^{p} \cdot
\end{gathered}
$$

Since

$$
\lim _{N \rightarrow \infty} \int_{1 /(N+s)}^{\infty}\left(\frac{1+s^{*}}{N+s+\alpha}+t\right)^{-(1+s) / p} d \sigma(t)=\int_{0}^{\infty} t^{-(1+s) / p} d \sigma(t),
$$

there exists an integer $N$ such that

$$
\begin{aligned}
\int_{1 /(N+s)}^{\infty} \frac{1}{t^{\alpha}}\left(\frac{1+s^{*}}{N+s+\alpha}+t\right)^{-(1+s) / p} d \sigma(t) & >(1-\eta) \int_{0}^{\infty} t^{-\alpha-(1+s) / p} d \sigma(t) \\
& =(1-\eta) C(p, s)
\end{aligned}
$$

Since

$$
\lim _{\varepsilon \rightarrow 0}\left(\frac{1+s^{*}}{N+s+\alpha}+t\right)^{-\varepsilon}=1
$$

there exists an $\varepsilon_{1}>0$ such that, for $0<\varepsilon<\varepsilon_{1}$,

$$
\begin{aligned}
& \int_{1 /(N+s)}^{\infty} \frac{1}{t^{\alpha}}\left(\frac{1+s^{*}}{N+s+\alpha}+t\right)^{-(1+s) / p-\varepsilon} d \sigma(t) \\
& \quad>(1-\eta) \int_{1 /(N+s)}^{\infty} \frac{1}{t^{\alpha}}\left(\frac{1+s^{*}}{N+s+\alpha}+t\right)^{-(1+s) / p} d \sigma(t)
\end{aligned}
$$

Finally,

$$
\sum_{n=0}^{\infty}(n+s+1)^{-(1+\varepsilon p)}=\sum_{n=0}^{N-1}(n+s+1)^{-(1+\varepsilon p)}+\sum_{n=N}^{\infty}(n+s+1)^{-(1+\varepsilon p)} .
$$

The ratio of $\sum_{n=0}^{\infty} / \sum_{n=N}^{\infty}$ tends to 1 as $\varepsilon \rightarrow 0$. Therefore there exists an $\varepsilon_{2}>0$ such that, for $0<\varepsilon<\varepsilon_{2}$,

$$
\sum_{n=N}^{\infty}(n+s+1)^{-(1+\varepsilon p)}>(1-\eta) \sum_{n=0}^{\infty}(n+s+1)^{-(1+\varepsilon p)} .
$$


Substituting (9)-(11) into (8) yields, for $\varepsilon=\min \left\{\varepsilon_{1}, \varepsilon_{2}\right\}$,

$$
\begin{aligned}
\|T a\|_{p, s}^{p} \geq & (1-\eta)^{p} \sum_{n=N}^{\infty}(n+\alpha+s+1)^{-(1+\varepsilon p)} \\
& \cdot\left[(1-\eta) \int_{1 /(N+s)}^{\infty} \frac{1}{t^{\alpha}}\left(\frac{1+s^{*}}{N+s+\alpha}+t\right)^{-(1+\varepsilon p)} d \sigma(t)\right]^{p} \\
\geq & (1-\eta)^{2 p} \sum_{n=N}^{\infty}(n+\alpha+s+1)^{-(1+\varepsilon p)}[(1-p) C(s, p)]^{p} \\
\geq & (1-\eta)^{3 p+1}(C(s, p))^{p} \sum_{n=0}^{\infty}(n+\alpha+s+1)^{-(1+\varepsilon p)} \\
= & (1-\eta)^{3 p+1}\left(C(s, p)^{p}\right)\|a\|_{p, s}^{p},
\end{aligned}
$$

and the finiteness of $C(p, s)$ is necessary for the norm of the operator $T$ to be finite. Moreover, $C(p, s)$ is the norm.

To prove (iii), suppose that $T$ is bounded and consider the transformation $T_{\delta}$ generated by restricting $\sigma$ to $[\delta, \infty)$ for some positive $\delta$.

From (a) $T_{\delta}$ is bounded. From (b) (ii),

$$
\left\|T_{\delta}\right\|=t^{-\alpha-(1+s) / p} d \sigma(t) .
$$

Moreover, $\left|\left(T_{\delta} a\right)(y)\right| \leq\left(T_{\delta}|a|\right)(y) \leq(T|a|)(y)$ for $y \geq 0$. Therefore $\left\|T_{\delta}\right\| \leq\|T\|$, which implies that $C(p, s) \leq\|T\|<\infty$ as $\delta \rightarrow 0$.

Corollary 1. For $s>-1,1 \leq p \leq \infty$,

$$
\int_{0}^{\infty}\left|\sum_{n=0}^{\infty} \frac{(y+\alpha)^{n+\alpha} e^{-(y+\alpha)}}{\Gamma(n+\alpha+1)} a_{n}\right|^{p}(y+\alpha)^{s} d y \leq \sum_{n=0}^{\infty}\left|a_{n}\right|^{p}(n+\alpha+1)_{s},
$$

where the constant is best possible, and for $1<p<\infty$ the strict inequality is valid unless the sequence $a$ is null.

Proof. In Theorem 1 choose the measure $\sigma$ to be the unit mass at $t=1$.

Define a new generalized Abel mean $A^{(\nu, \alpha)}(x)$ of order $\nu$, involving the parameter $\alpha \geq 0$, of a sequence $\left\{a_{n}\right\}$, and the modified moments $A_{n}^{(\nu, \alpha)}, \nu>0, \alpha \geq 0$, of a function $a(x), \alpha /(1+\alpha) \leq x \leq 1$, by

$$
\begin{aligned}
A^{(\nu, \alpha)}(x) & =\frac{(1-x)^{\nu}}{\Gamma(\alpha+1)} \sum_{n=0}^{\infty} \frac{\left(\begin{array}{c}
n+\nu+\alpha-1 \\
\nu+\alpha-1
\end{array}\right)}{\left(\begin{array}{c}
n+\alpha \\
\alpha
\end{array}\right)} a_{n} x^{n+\alpha}, \\
A_{n}^{(\nu, \alpha)} & =\frac{1}{\Gamma(\alpha+1)} \frac{\left(\begin{array}{c}
n+\nu+\alpha-1 \\
\nu+\alpha-1
\end{array}\right)}{\left(\begin{array}{c}
n+\alpha \\
\alpha
\end{array}\right)} \int_{\frac{\alpha}{1+\alpha}}^{1} x^{n+\alpha} a(x) d x .
\end{aligned}
$$

For $\alpha=0$, these reduce to the standard generalized Abel means and moments. If also $\nu=1$, then one has the ordinary Abel means and moments.

Corollary 2. For $1<p<\infty, \nu>0, \alpha \geq 0$, and $-1<s<\nu p-1$,

(a) $\begin{aligned} \int_{\frac{\alpha}{1+\alpha}}^{1} & \left|A^{(\nu, \alpha)}(x)\right|^{p}(1-x)^{-s-2} x^{s} d x \\ < & {\left[\frac{\Gamma((p \nu-s-1) / p)}{\Gamma(\nu+\alpha)}\right]^{p} \sum_{n=0}^{\infty}\left|a_{n}\right|^{p}(n+\alpha+1)_{s}, }\end{aligned}$ 


$$
\text { (b) } \begin{aligned}
\sum_{n=0}^{\infty} \mid & \left.A_{n}^{(\nu, \alpha)}\right|^{p}(n+\alpha+1)_{s}^{1-p} \\
& <\left[\frac{\Gamma\left(\left(p^{\prime} \nu-s-1\right) / p^{\prime}\right)}{\Gamma(\nu+\alpha)}\right]^{p} \int_{\frac{\alpha}{1+\alpha}}^{1}|a(x)|^{p}(1-x)^{(2-\nu) p-s-2} x^{s+\alpha} d x,
\end{aligned}
$$

and, for $1<p<\infty$, strict inequality is valid unless the sequence $\left\{a_{n}\right\}$ is null, or $a(x)$ is null a.e., and the constants are best possible.

Proof. For (a) use the gamma probability density $d \sigma(t)=t^{\nu+\alpha-1} e^{-t} d t / \Gamma(\nu+\alpha)$. Then

$$
\begin{gathered}
C(p, s)=\frac{\Gamma((p \nu-s-1) / p)}{\Gamma(\nu+\alpha)} \text { for }-1<s<\nu p-1, \\
(T a)(y)=\int_{0}^{\infty} \sum_{n=0}^{\infty} \frac{a_{n}(t(y+\alpha))^{n+\alpha}}{t^{\alpha} \Gamma(n+\alpha+1)} e^{-t(y+\alpha)} \frac{t^{\nu+\alpha-1} e^{-t}}{\Gamma(\nu+\alpha)} d t \\
=\sum_{n=0}^{\infty} \frac{a_{n}(y+\alpha)^{n+\alpha}}{\Gamma(n+\alpha+1) \Gamma(\nu+\alpha)} \int_{0}^{\infty} t^{n+\nu+\alpha-1} e^{-t(y+\alpha+1)} d t \\
=\sum_{n=0}^{\infty} \frac{a_{n}(y+\alpha)^{n+\alpha}}{\Gamma(n+\alpha+1) \Gamma(\nu+\alpha)} \frac{\Gamma(n+\nu+\alpha)}{(y+\alpha+1)^{n+\nu+\alpha}} \\
=\frac{(y+\alpha+1)^{-\nu}}{\Gamma(\alpha+1)} \sum_{n=0}^{\infty} \frac{\left(\begin{array}{c}
n+\nu+\alpha-1 \\
\nu+\alpha-1
\end{array}\right)}{\left(\begin{array}{c}
n+\alpha \\
\alpha
\end{array}\right)}\left(\frac{y+\alpha}{y+\alpha+1}\right)^{n+\alpha} .
\end{gathered}
$$

Now set $x=(y+\alpha) /(y+\alpha+1)$. Then $[0, \infty)$ gets mapped onto $[\alpha /(1+\alpha), 1)$, $(y+\alpha)^{s} d y=x^{s}(1-x)^{-s-2} d x$, and the result follows from Theorem 1.

To prove (b), let $\sigma$ be a finite positive measure such that $C\left(p^{\prime}, s\right)<\infty$. Then the transformation $T^{*}$ for $f \in L^{p, s}[0, \infty)$, given by

$$
\left(T^{*} f\right)_{n}=\int_{0}^{\infty} f(y)(y+\alpha)^{s} d y \int_{0}^{\infty} \frac{e^{-t(y+\alpha)}(t(y+\alpha))^{n+\alpha} d \sigma(t)}{t^{\alpha} \Gamma(n+s+\alpha+1)},
$$

is the adjoint of the transformation $T$ in Theorem 1 . Therefore $T^{*}$ is a bounded transformation from $L^{p, s}[0, \infty)$ into $\ell^{p, s}$, and $\left\|T^{*} f\right\|_{p, s} \leq C\left(p^{\prime}, s\right)\|f\|_{p, s}$ unless $f$ is null a.e. Let $\sigma$ be the gamma probability density function from part (a). Then

$$
C\left(p^{\prime}, s\right)=\left[\frac{\Gamma\left(\left(p^{\prime} \nu-s-1\right) / p\right)}{\Gamma(\nu+\alpha)}\right]^{p},
$$

and

$$
\begin{aligned}
\left(T^{*} f\right)_{n}= & \frac{(n+\alpha+1)_{s}^{-1}}{\Gamma(\alpha+1)} \frac{\left(\begin{array}{c}
n+\nu+\alpha-1 \\
\nu+\alpha-1
\end{array}\right)}{\left(\begin{array}{c}
n+\alpha \\
\alpha
\end{array}\right)} \\
& \cdot \int_{0}^{\infty}\left(\frac{y+\alpha}{y+n+\alpha}\right)^{n+\alpha} f(y)(y+\alpha)^{s}(y+\alpha+1)^{-\nu} d y .
\end{aligned}
$$

Let $A_{n}^{(\nu, \alpha)}$ be the generalized moments as defined above, for the function $a(x)$, $\alpha /(1+\alpha) \leq x<1$. Make the change of variable $y+\alpha+1=1 /(1-x)$, which maps 
$[\alpha /(1+\alpha), 1)$ onto $[0, \infty)$ so that $d y=d x /(1-x)^{2}$ and $(y+\alpha)^{s} d y=(1-x)^{-s-2} x^{s} d x$. Then

$$
A_{n}^{(\nu, \alpha)} /(n+\alpha+1)_{s}=\left(T^{*} f\right)_{n}
$$

where $f(y)=(y+\alpha+1)^{\nu-\alpha} a((y+\alpha) /(y+\alpha+1))$.

Theorem 2. Consider the transformation $T_{\lambda}$ defined by

$$
\left(T_{\lambda} a\right)(y):=(y+\alpha)^{1-\lambda} \sum_{n=0}^{\infty} a_{n} \int_{0}^{\infty} \frac{e^{-t(y+\alpha)}(t(y+\alpha))^{n+\alpha}}{t^{\alpha} \Gamma(n+\alpha+1)} \Psi(t) d t
$$

where

$$
C(\lambda, p, r):=\int_{0}^{\infty} t^{-(\alpha+1 / r) / \lambda}|\Psi(t)|^{1 / \lambda} d t, \quad 1 / r=1 / p+\lambda-1 .
$$

Then $T_{\lambda}$ is bounded transformation from $\ell^{p}$ to $L^{p}[0, \infty)$ for $1<p<r<\infty$, and $\left\|T_{\lambda} a\right\|_{r} \leq C(\lambda, p, r)^{\lambda}\|a\|_{p}$. Moreover, if $\Psi \geq 0$, then $\left\|T_{\lambda} a\right\|_{r}<C(\lambda, p, r)^{\lambda}\|a\|_{p}$ unless $a$ is a null sequence.

Proof. Let $f \in L^{r^{\prime}}[0, \infty), 0<\lambda<1,1<p<r<\infty$. Let $E_{t}(a)$ be as in Theorem 1 . Then, using Hölder's inequality with $1 / r+1 / p^{\prime}+1-\lambda=1$,

$$
\begin{aligned}
\left|\int_{0}^{\infty}\left(T_{\lambda} a\right)(y) f(y) d y\right| \leq & \int_{0}^{\infty} \int_{0}^{\infty}\left|\left(E_{t} a\right)(y)\right|(y+\alpha)^{1-\lambda}|f(y)| d y \\
= & \int_{0}^{\infty} \int_{0}^{\infty}\left[\left|t^{\alpha}\left(E_{t} a\right)(y)\right| t^{-\left(\alpha-1 / p^{\prime}\right) / \lambda}|\Psi(t)|^{1 / \lambda}\right]^{1 / r} \\
& \left.\cdot\left[|f(y)|^{r^{\prime}} t^{-(\alpha+1 / r) / \lambda}|\Psi(t)|^{1 / \lambda}\right]\right]^{1 / p^{\prime}} \\
& \cdot\left[\left|t^{\alpha}\left(E_{t} a\right)(y)\right|^{p}|p(y)|^{r^{\prime}}(y+\alpha)\right]^{1-\lambda} d t d y \\
\leq & {\left[\int_{0}^{\infty} \int_{0}^{\infty}\left|t^{\alpha}\left(E_{t} a\right)(y)\right|^{p} t^{-\left(\alpha-1 / p^{\prime}\right) / \lambda}|\Psi(t)|^{1 / \lambda} d t d y\right]^{1 / r} } \\
& \cdot\left[\int_{0}^{\infty} \int_{0}^{\infty}|f(y)|^{r^{\prime}} t^{-(\alpha+1 / r) / \lambda}|\Psi(t)|^{1 / \lambda} d t d y\right]^{1 / p^{\prime}} \\
= & P^{1 / r} Q^{1 / p^{\prime}} R^{1-\lambda} .
\end{aligned}
$$


Using (6) and Hölder's inequality,

$$
\begin{aligned}
P & \leq \int_{0}^{\infty} \int_{0}^{\infty} t^{-\left(\alpha-1 / p^{\prime}\right) / \lambda}|\Psi(t)|^{1 / \lambda} \sum_{n=0}^{\infty}\left|a_{n}\right|^{p} \frac{e^{-t(y+\alpha)}(t(y+\alpha))^{n+\alpha}}{\Gamma(n+\alpha+1)} d t d y \\
& =\int_{0}^{\infty} t^{-\left(\alpha-1 / p^{\prime}\right) / \lambda}|\Psi(t)|^{1 / \lambda} \sum_{n=0}^{\infty} \frac{\left|a_{n}\right|^{p}}{\Gamma(n+\alpha+1)} \int_{0}^{\infty} e^{-t(y+\alpha)}(t(y+\alpha))^{n+\alpha} d y d t \\
& \leq \int_{0}^{\infty} t^{-\left(\alpha-1 / p^{\prime}\right) / \lambda}|\Psi(t)|^{1 / \lambda} \sum_{n=0}^{\infty} \frac{\left|a_{n}\right|^{p}}{\Gamma(n+\alpha+1)} \cdot \frac{\Gamma(n+\alpha+1)}{t} d t \\
& =\int_{0}^{\infty} t^{-(\alpha+1 / r) / \lambda}|\Psi(t)|^{1 / \lambda}\|a\|_{p}^{p} d t=C(\lambda, p, r)\|a\|_{p}^{p}, \\
Q & =\int_{0}^{\infty} \int_{0}^{\infty}|f(y)|^{r^{\prime}} t^{-(\alpha+1 / r) / \lambda}|\Psi(t)|^{1 / \lambda} d t \\
& =C(\lambda, p, r)\|f\|_{r^{\prime}}^{r^{\prime}},
\end{aligned}
$$

and

$R \leq\|f\|_{r^{\prime}}^{r^{\prime}}\|a\|_{p}^{p}$.

Therefore

$$
\left|\int_{0}^{\infty}\left(T_{\lambda} a\right)(y) f(y) d y\right| \leq C(\lambda, p, r)^{\lambda}\|a\|_{p}\|f\|_{r^{\prime}}
$$

and

$$
\left\|T_{\lambda} a\right\|_{r} \leq C(\lambda, p, r)^{\lambda}\|a\|_{p} .
$$

Corollary 3. If $1<p<r<\infty, 1 / r=1 / p+\lambda-1, \nu>\alpha+1 / p$, then

$$
\begin{aligned}
{\left[\int_{\frac{\alpha}{1+\alpha}}^{1}\left|A^{(\nu, \alpha)}(x)\right|^{r}(1-\right.} & \left.x)^{-r / p-1} x^{r / p-1} d x\right]^{1 / p} \\
& <\frac{1}{\Gamma(\nu+\alpha)}\left[\lambda^{(\nu p-1) / \lambda p} \Gamma((\nu p-1) / p)\right]^{\lambda}\left[\sum_{n=0}^{\infty}\left|a_{n}\right|^{p}\right]^{1 / p} .
\end{aligned}
$$

unless the sequence $a$ is null.

Proof. In Theorem 2 replace $\Psi$ with $\left[t^{\nu+\alpha-1} e^{-t} / \Gamma(\nu+\alpha)\right]$. One immediately obtains the left-hand side of (12), after changing the variable from $y$ in terms of $x$.

$$
\begin{aligned}
C(\lambda, p, r) & =\frac{1}{\Gamma(\nu+\alpha)^{1 / \lambda}} \int_{0}^{\infty} t^{-(\alpha+1 / r) / \lambda}\left(t^{\nu+\alpha-1} e^{-t}\right)^{1 / \lambda} d t \\
& =\frac{1}{\Gamma(\nu+\alpha)^{1 / \lambda}} \int_{0}^{\infty} e^{-t / \lambda} t^{(\nu-1-1 / r) / \lambda} d t \\
& =\frac{\lambda^{(\nu p-1) / \lambda p} \Gamma((\nu p-1) / p \lambda)}{\Gamma(\nu+\alpha)} .
\end{aligned}
$$

Define

$$
A^{(\alpha)}(x)=\frac{\Gamma(\alpha+1) A^{(1, \alpha)}(x)}{(1-x)}, \quad A_{n}^{(\alpha)}=\Gamma(\alpha+1) A_{n}^{(1, \alpha)} .
$$


Theorem 3. For $1 \leq p<r<\infty, q>0$, we have

$$
\begin{gathered}
\text { (a) }\left[\int_{\frac{\alpha}{1+\alpha}}^{1}\left|\sum_{n=0}^{\infty} a_{n} x^{n+\alpha}\right|^{r}(1-x)^{r / q-1} d x\right]^{1 / r} \\
<K(p, r, q)\left[\sum_{n=0}^{\infty}\left|a_{n}\right|^{p}(n+1)^{-(p+q-p q) / q}\right]^{1 / p}, \\
\text { (b) }\left[\sum_{n=0}^{\infty}\left|\int_{\frac{\alpha}{1+\alpha}}^{1} x^{n+\alpha} a(x) d x\right|^{r}(n+1)^{r-1}\right]^{1 / r} \\
<K\left(r^{\prime}, p^{\prime}, q\right)\left[\int_{\frac{\alpha}{1+\alpha}}^{1}|a(x)|^{p}(1-x)^{-(p+q-p q) / q} d x\right]^{1 / p},
\end{gathered}
$$

unless the sequence $a_{n}$ is null or $a(x)$ is null a.e., respectively, and where

$$
\begin{aligned}
& K(p, q, r)^{r}=\max \left(\Gamma\left(p^{\prime} / q\right), 1\right)^{(r-p) / p^{\prime}} \\
& \cdot[\max (\Gamma(\alpha+1), 1) \max (1 / \Gamma(\nu+\alpha), 1) \Gamma(1 / q)]^{p} .
\end{aligned}
$$

Moreover, the constant in (a) is best possible for $r=p, q \geq p^{\prime}$, and in (b) for $r=p, q \geq p, 1-1 / p-1 / q \leq \alpha \leq 1$.

Lemma $2\left(\left[2\right.\right.$, Lemma 2]). If $C_{n \nu}:=\left(\begin{array}{c}n+\nu-1 \\ \nu-1\end{array}\right), n$ is an integer and $\nu$ is real, then we have

(a) $C_{n \nu} \geq(n+1)^{\nu-1} / \max (\Gamma(\nu), 1)$ for $n \geq 0, \nu>0$,

(b) $C_{n \nu} \leq(n+1)^{\nu-1} / \min (\Gamma(\nu), 1)$ for $n \geq 0, \nu>1$,

(c) $C_{n \nu} \leq n^{\nu-1} / \Gamma(\nu)$ for $n \geq 1,0<\nu \leq 1$.

Proof. For $p=r, \nu=1 / p+1 / q, s=0$, we have, from Corollary 2(a),

$$
\int_{\frac{\alpha}{1+\alpha}}^{1}\left|A^{(\nu, \alpha)}(x)\right|^{p}(1-x)^{-2} d x<\left[\frac{\Gamma((p \nu-1) / p)}{\Gamma(\nu+\alpha)}\right]^{p} \sum_{n=0}^{\infty}\left|a_{n}\right|^{p} .
$$

With $a_{n}=\left|a_{n}\right|(n+1)^{1-\nu}$,

$$
A^{(v, \alpha)}(x)=\frac{(1-x)^{v}}{\Gamma(\alpha+1)} \sum_{n=0}^{\infty} \frac{\left(\begin{array}{c}
n+\nu+\alpha-1 \\
\nu+\alpha-1
\end{array}\right)}{\left(\begin{array}{c}
n+\alpha \\
\alpha
\end{array}\right)}\left|a_{n}\right|(n+1)^{1-\nu} x^{n+\alpha} .
$$

From Lemma 2(a),

$$
\left(\begin{array}{c}
n+\nu+\alpha-1 \\
\nu+\alpha-1
\end{array}\right) \geq \frac{(n+1)^{\nu+\alpha-1}}{\max (\Gamma(\nu+\alpha), 1)}
$$

From Lemma 2(b),

$$
\frac{1}{\left(\begin{array}{c}
n+\alpha \\
\alpha
\end{array}\right)} \geq \frac{\min (\Gamma(\alpha+1), 1)}{(n+1)^{\alpha}}
$$

Thus

$$
\left|A^{(\nu, \alpha)}(x)\right| \geq \frac{(1-x)^{\nu} \min (\Gamma(\alpha+1), 1) A^{(\alpha)}(x)}{\max (\Gamma(\nu+\alpha), 1) \Gamma(\alpha+1)}
$$

But

$$
\frac{\min (\Gamma(\alpha+1), 1)}{\Gamma(\alpha+1)}=\min (1 / \Gamma(\alpha+1), 1)=\max (\Gamma(\alpha+1), 1)
$$


Substituting into (13) yields

$$
\begin{aligned}
{\left[\int_{\frac{\alpha}{\alpha+1}}^{1}\left|A^{(\alpha)}(x)\right|^{p}(1-x)^{p / q-1} d x\right]^{1 / p} } \\
<\max (\Gamma(\alpha+1), 1) \max (1 / \Gamma(\nu+\alpha), 1) \Gamma((p \nu-1) / p) \\
\cdot\left[\sum_{n=0}^{\infty}\left|a_{n}\right|^{p}(n+1)^{-(p+q-p q)}\right]^{1 / p} \cdot
\end{aligned}
$$

For the general case, with $C:=p^{\prime} / q, \nu=1 / p+1 / q$ we have, using Lemma 2(a),

$$
\begin{aligned}
\left|A^{(\alpha)}(x)\right| & \leq \sum_{n=0}^{\infty}\left|a_{n}\right| x^{n+\alpha} \\
& =\sum_{n=0}^{\infty}(n+1)^{1-\nu}\left|a_{n}\right|(n+1)^{\nu-1} x^{n+\alpha} \\
& \leq\left[\sum_{n=0}^{\infty}(n+1)^{p(1-\nu)}\left|a_{n}\right|^{p} x^{n+\alpha}\right]^{1 / p} \\
& \cdot\left[\sum_{n=0}^{\infty}(n+1)^{(\nu-1) p^{\prime}} x^{n+\alpha}\right]^{1 / p^{\prime}} \\
& \leq S_{p} \max (\Gamma(c), 1)^{1 / p^{\prime}}\left[\sum_{n=0}^{\infty}(n+c-1) x^{n}\right]^{1 / p^{\prime}} \\
& =\max (\Gamma(c), 1)^{1 / p^{\prime}}(1-x)^{-c} S_{p},
\end{aligned}
$$

where

$$
\begin{gathered}
S_{p}=\sum_{n=0}^{\infty}(n+1)^{p(1-\nu)}\left|a_{n}\right|^{p}, \\
\int_{\frac{\alpha}{\alpha+1}}^{1}\left|A^{(\alpha)}(x)\right|^{r}(1-x)^{r / q-1} d x=\int_{\frac{\alpha}{\alpha+1}}^{1}\left|A^{(\alpha)}(x)\right|^{r-p}\left|A^{\alpha)}(x)\right|^{p}(1-x)^{r / q-1} d x \\
\leq(\max (\Gamma(c), 1))^{\frac{r-p}{p^{\prime}}} S_{p}^{r-p} \int_{\frac{\alpha}{\alpha+1}}^{1}\left|A^{(\alpha)}(x)\right|^{p}(1-x)^{p / q-1} d x \\
\leq(\max (\Gamma(c), 1))^{(r-p) / p^{\prime}} S_{p}^{r-p}[\max (\Gamma(\alpha+1), 1) \max (1 / \Gamma(\nu+\alpha), 1) \Gamma(1 / q)]^{p} S_{p}^{p},
\end{gathered}
$$

and (a) is proved.

Part (b) is derived from part (a) by the same duality argument that Hardy and Littlewood use in [3] to prove Theorem 4 from Theorem 3. In fact a duality argument yields (a) from (b). Therefore, to show that the constant is best possible, it is sufficient to show that the constant is best possible in (a) when $r=p$ and $q \leq p^{\prime}$, in which case the constant is $k \Gamma(1 / q)$, where

$$
k:=\max (\Gamma(\alpha+1), 1) \max (1 / \Gamma(1 / p+1 / q+\alpha), 1) .
$$


Note that $k \geq 1$. When $0 \leq \alpha \leq 1$ and $1 \leq 1 / p+1 / q+\alpha \leq 2$, i.e., $1-1 / p-1 / q \leq$ $\alpha \leq 1, k=1$.

With $k=1$, for $0<\varepsilon<1 / q<1$, define $\left\{a_{n}\right\}$ by $a_{0}=\Gamma(\nu), a_{n}=n^{\nu-1}, n>0$, where $\nu:=1 / q-\varepsilon$.

Using the same argument as the one on page 54 of [2],

$$
\begin{aligned}
S_{p}^{p}-\Gamma(\nu)^{p} & =\sum_{n=1}^{\infty} n^{p(\nu-1)}(n+1)^{-(p+q-p q) / q} \\
& \leq \frac{1}{\varepsilon p}+0(1),
\end{aligned}
$$

which implies that

$$
\frac{1}{\varepsilon p} \geq \frac{S_{p}^{p}}{1+o(1)}
$$

as $\varepsilon \rightarrow 0$, where $o(1)>0$. Using Lemma $2(\mathrm{c})$,

$$
\begin{aligned}
\left|A^{(\alpha)}(x)\right| & =x^{\alpha}\left[\Gamma(\nu)+\sum_{n=1}^{\infty} \eta^{\nu-1} x^{n}\right] \\
& \geq x^{\alpha} \Gamma(\nu)\left[1+\sum_{n=1}^{\infty}\left(\begin{array}{c}
n+\nu-1 \\
\nu-1
\end{array}\right) x^{n}\right] \\
& =\Gamma(\nu) x^{\alpha}(1-x)^{-\nu} .
\end{aligned}
$$

Then

$$
\begin{aligned}
\int_{\frac{\alpha}{\alpha+1}}^{1} & \left|A^{(\alpha)}(x)\right|^{p}(1-x)^{p / q-1} d x \geq \Gamma(\nu)^{p} \int_{\frac{\alpha}{\alpha+1}}^{1} x^{p \alpha}(1-x)^{p \varepsilon-1} d x \\
& =\Gamma(\nu)^{p}\left[\int_{0}^{1} x^{p \alpha}(1-x)^{p \varepsilon-1} d x-\int_{0}^{\frac{\alpha}{1+\alpha}} x^{p \alpha}(1-x)^{p \varepsilon-1} d x\right] \\
& \geq \Gamma(\nu)^{p}\left[\frac{\Gamma(p \alpha+1) \Gamma(p \varepsilon)}{\Gamma(p \alpha+p \varepsilon+1)}-\frac{1}{p \varepsilon}\left(\frac{\alpha}{1+\alpha}\right)^{p \alpha}\left(1-\frac{1}{(\alpha+1)^{p \varepsilon}}\right)\right] \\
& =\frac{\Gamma(\nu)^{p}}{p \varepsilon}\left[\frac{\Gamma(p \alpha+1) \Gamma(p \varepsilon+1)}{\Gamma(p \alpha+p \varepsilon+1)}-\left(\frac{\alpha}{1+\alpha}\right)^{p \alpha}\left(1-\frac{1}{(\alpha+1)^{p \varepsilon}}\right)\right] .
\end{aligned}
$$

The quantity in brackets is $1-o(1)$ as $\varepsilon \rightarrow 0$. Using (15),

$$
\int_{\frac{\alpha}{\alpha+1}}^{1}\left|A^{(\alpha)}(x)\right|^{p}(1-x)^{p / q-1} d x \geq \frac{\Gamma(\nu)^{p}}{p \varepsilon}(1-o(1)) \geq \Gamma(1 / q)^{p} \frac{(1-o(1)) S_{p}^{p}}{1+(o(1))}
$$

as $\varepsilon \rightarrow 0$.

It is an open question whether $k \Gamma(1 / q)$ is the best constant when $k>1$. 
The analogues to inequalities $A_{0}, B_{0}, C_{0}$, and $D_{0}$ in [2] are

$$
\begin{aligned}
& \left(A_{0}\right) \int_{\frac{\alpha}{1+\alpha}}^{1}\left|\sum_{n=0}^{\infty} a_{n} x^{n+\alpha}\right|^{p}(1-x)^{(1-\alpha) p-2} d x<\left[\frac{\Gamma(\alpha+1) \Gamma\left(1 / p^{\prime}\right)}{\Gamma(\nu+\alpha)}\right]^{p} \sum_{n=0}^{\infty}\left|a_{n}\right|^{p}, \\
& \left(B_{0}\right) \int_{\frac{\alpha}{\alpha+1}}^{1}\left|\sum_{n=0}^{\infty} a_{n} x^{n+\alpha}\right|^{p} d x<[\max (\Gamma(\alpha+1), 1)(1 / \Gamma(2 / p+\alpha), 1) \Gamma(1 / p)]^{p} \\
& \sum_{n=0}^{\infty}\left|a_{n}\right|^{p}(n+1)^{p-2} \\
& \left(C_{0}\right) \sum_{n=0}^{\infty}\left|\int_{\frac{\alpha}{\alpha+1}}^{1} x^{n+\alpha} a(x) d x\right|^{p}<\left[\max (\Gamma(\alpha+1), 1) \max \left(1 / \Gamma\left(2 / p^{\prime}+\alpha\right), 1\right) \Gamma\left(1 / p^{\prime}\right)\right]^{p} \\
& \int_{\frac{\alpha}{\alpha+1}}^{1}|a(x)|^{p}(1-x)^{p-2} d x \\
& \left(D_{0}\right) \sum_{n=0}^{\infty}\left|\int_{\frac{\alpha}{\alpha+1}}^{1} x^{n+\alpha} a(x) d x\right|^{p}<\left[\frac{\Gamma(\alpha+1) \Gamma(1 / p)}{\Gamma\left(2 / p^{\prime}+\alpha\right)}\right]^{p} \int_{\frac{\alpha}{\alpha+1}}^{1}|a(x)|^{p}(1-x)^{p-2} x^{\alpha} d x .
\end{aligned}
$$

$\left(A_{0}\right)$ and $\left(D_{0}\right)$ are obtained from Corollary 2 by setting $v-1, s=0$. Similarly, $\left(B_{0}\right)$ and $\left(C_{0}\right)$ are obtained from Theorem 3 by setting $r=p, q=p$ in (a), and $r=p, q=p^{\prime}$ in (b).

Setting $\alpha=0$ in the results of this paper yields the corresponding results from $[2]$.

\section{REFERENCES}

1. K. Endl, Untersuchungen über Momentprobleme bei Verfahren von Hausdorffschen Typus, Math. Annal. 139 (1960), 403-432. MR 22:12329

2. C. Georgakis, Bounded sequence-to-function Hausdorff transformations, Proc. Amer. Math. Soc. 103 (1988), 531-542. MR 89i: 40003

3. G. H. Hardy and J. E. Littlewood, Elementary theorems concerning power series and moment constants, J. für Reine und Ang. Math. 157 (1927), 141-158.

4. A. Jakimovski, The product of summability methods; new classes of transformations and their properties, Contract No. Air Force 61(1052)-187(1959), 1-76.

Department of Mathematics, Indiana University, Bloomington, Indiana 47405-5701

E-mail address: rhoades@indiana.edu 